\title{
Exploring the Synthesis of Mesoporous Stannosilicates as Catalysts for the Conversion of Mono- and Oligosaccharidesinto Methyl Lactate
}

Tosi, Irene; Sacchetti, Annalisa; Martinez-Espin, Juan S.; Meier, Sebastian; Riisager, Anders

Published in:

Topics in Catalysis

Link to article, DOI:

$10.1007 / \mathrm{s} 11244-019-01135-8$

Publication date:

2019

Document Version

Peer reviewed version

Link back to DTU Orbit

Citation (APA):

Tosi, I., Sacchetti, A., Martinez-Espin, J. S., Meier, S., \& Riisager, A. (2019). Exploring the Synthesis of Mesoporous Stannosilicates as Catalysts for the Conversion of Mono- and Oligosaccharidesinto Methyl Lactate. Topics in Catalysis, 62(7-11), 628-638. https://doi.org/10.1007/s11244-019-01135-8

\section{General rights}

Copyright and moral rights for the publications made accessible in the public portal are retained by the authors and/or other copyright owners and it is a condition of accessing publications that users recognise and abide by the legal requirements associated with these rights.

- Users may download and print one copy of any publication from the public portal for the purpose of private study or research.

- You may not further distribute the material or use it for any profit-making activity or commercial gain

- You may freely distribute the URL identifying the publication in the public portal 


\title{
Exploring the Synthesis of Mesoporous Stannosilicates as Catalysts for the Conversion of Mono- and Oligosaccharides into Methyl Lactate
}

\author{
Irene Tosi ${ }^{1 \mathrm{a}}$, Annalisa Sacchetti ${ }^{2}$, Juan S. Martinez-Espin ${ }^{3}$, Sebastian Meier ${ }^{1 \mathrm{~b}}$ and Anders Riisager ${ }^{1 *}$ \\ ${ }^{1}$ Technical University of Denmark, Department of Chemistry, Kemitorvet, 2800-Kgs. Lyngby, Denmark \\ ${ }^{2}$ Dipartimento di Chimica Industriale "Toso Montanari”, Alma Mater Studiorum- University of Bologna, Viale del \\ Risorgimento 4, 40136, Bologna, Italy \\ ${ }^{3}$ Haldor Topsøe A/S, Haldor Topsøes Alle 1, 2800-Kgs. Lyngby, Denmark
}

${ }^{\mathrm{a} O R C I D ~ I D: ~ 0000-0002-2486-6971 . ~}$

'ORCID ID: 0000-00033761-3217.

*ar@kemi.dtu.dk, ORCID ID: 0000-0002-7086-1143.

\begin{abstract}
Sn-Beta zeolites are among the most promising catalysts for the conversion of biomasses due to their high Lewis acidity, which allows coordination to functionalized molecules and promotes cleavage and rearrangement reactions. For applications in biorefining zeolite porosity would ideally be optimized to avoid diffusional limitations, which otherwise may decrease reaction rates and restrict the conversion of bulky substrates. The synthesis of mesoporous zeolites can help alleviating limitations and is a central topic in heterogeneous catalysis, with many synthetic procedures for mesoporous zeolites proposed over the last decades. Here, we explore different syntheses routes to prepare Lewis acidic Sn-containing zeolites, and the main features of the prepared mesoporous materials are characterized. We investigate the correlation between different types of porosity and the activity for the conversion of sugars into methyl lactate. The monomer glucose, the dimer sucrose and the oligomer inulin are applied as model substrates for the reaction in order to probe the accessibility of molecules with different sizes to active sites in zeolites with different pore systems.
\end{abstract}

Keywords

biomass conversion, inulin, mesoporous zeolites,

methyl lactate, Sn-Beta 


\section{Introduction}

Sustainable production of fuels and chemicals requires the replacement of fossil feedstocks with renewable resources. Among those, abundant low cost biomass feedstock composed of highly functionalized molecules promises a favorable carbon footprint and the ability for transformation into many relevant chemicals along several chemo-catalytic routes [1].

In the last two decades, Lewis acidic zeotype materials have received much attention for biomass conversion due to their high Lewis acidity, which allows the coordination to functionalized molecules and promotes cleavage and rearrangement reactions $[2,3]$. Among the Lewis acidic zeotype materials, Snbeta zeolite is perhaps the most studied catalyst due to its versatile catalytic activity in the conversion of simple sugars, promoting transformations such as isomerization [4, 5] and epimerization [6] of glucose, retro-aldol reactions [3], hydride-shifts and rearrangements into different products [7]. The production of methyl lactate starting from either glucose, fructose or sucrose has arguably remained the most important process catalyzed by Sn-Beta, as this process permits the sustainable production of the monomer for polylactic acid (PLA) formation [8]. PLAs represent about $10 \%$ of the raw material globally produced for bioplastics, and their production capacity is expected to grow by $50 \%$ by 2022 as a consequence of the biobased origin and biodegradability of the compounds [9]. Additional $\alpha$-hydroxy esters, such as methyl vinyl glycolate (MVG) and methyl trans-2,5,6trihydroxy-3-hexenoate (THM) are formed in the sugar conversion reaction, making the process a possible source for the production of diverse polyester monomers that contains additional groups for functionalization $\quad[7,10-12] \quad$ (Figure 1).<smiles>[R]OC(=O)[C@@H](O)/C=C/[C@@H](O)CO</smiles>

THM<smiles>C=C[C@H](O)C(=O)O[Na]</smiles>

MVG

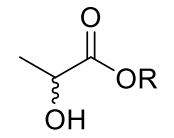

$\mathrm{ML}$
Figure 1. Bio-based $\alpha$-hydroxy esters as monomers deriving in the process converting simple sugars with Sn-Beta zeolite catalysts.

Although Sn-Beta bears great potential as a catalyst for biorefineries, it has been applied only to the conversion of simple sugars, while applications to the conversion of second generation biomass components are still lacking [13]. Biomass components are often oligo- or polysaccharides that meet diffusional limitations when they are converted with microporous catalysts such as zeolites [14]. For this reason, the preparation of hierarchical materials that permit increased substrate diffusion rates and can host larger molecules than microporous catalysts is a central issue in the field of heterogeneous catalysis [15-17]. Furthermore, catalyst deactivation promoted by accumulation of coke inside the pores has a smaller impact in large pore systems than in microporous materials, with beneficial impact on the catalyst lifetime [18]. Synthetic procedures for the preparation of mesoporous Sn-beta zeolites have been considered in several studies [19-22], but the control of mesopore insertion and the suitability for the conversion of oligosaccharides have remained challenging.

Here, we investigate the catalytic performance of different mesoporous Sn-zeolitic materials for sugar conversion. Utilizing two selected zeolite frameworks, USY and BEA, several synthetic procedures were explored for the preparation of mesoporous Sn-derived materials, and the resulting materials were studied in the conversion of different sized carbohydrates. Glucose, sucrose and inulin were used as substrates to investigate if loss of reactivity accompanied the formation of the mesopores.

\section{Results and discussion}

\subsection{Screening of different zeolite frameworks}

Microporous stannosilicates have previously been prepared following a post-synthetic route [23]. Starting from commercially available zeolites, the procedure involves an acidic treatment to remove aluminum, followed by the introduction of $\mathrm{Sn}$ by incipient wetness impregnation of an aqueous solution of the metal precursor. Post-synthetic procedures present several advantages compared to hydrothermal synthesis such as larger preparation scales, short crystallization times and the avoidance of $\mathrm{HF}$ as crystallization agent [24].

Initially, different zeolite frameworks were evaluated as starting materials for the post-synthetic methodology. Several zeolite frameworks were resistant to dealumination and did not offer the possibility for post-synthetic modifications. Frameworks BEA, USY, MOR, and MFI were subjected to dealumination treatments. Among these frameworks, only BEA and USY materials resulted in an efficient dealumination after treatment overnight in $\mathrm{HNO}_{3}(13 \mathrm{M})$ at $100{ }^{\circ} \mathrm{C}$. Moreover, different $\mathrm{Si} / \mathrm{Al}$ ratios in the starting zeolite did not seem to have a significant effect on the dealumination process (Supporting Information Table S1). Accordingly, HUSY (6) and H-USY (30) possessed similar Al content 
after acidic treatment and formed materials with similar catalytic activity after Sn impregnation. However, zeolites USY are stable materials and resulted in low $\mathrm{Si} / \mathrm{Al}$ ratio after the acidic treatment (SI). Following an initial screening, the microporous catalysts $[\mathrm{deAl}] \mathrm{Sn}-\mathrm{BEA}(\mathrm{Si} / \mathrm{Sn}=100)$ and $[\mathrm{deAl}] \mathrm{Sn}-$ USY ( $\mathrm{Si} / \mathrm{Sn}=25)$ showed the best catalytic activity in the conversion of glucose to methyl lactate. Hence, [deAl] Sn-BEA (100) and [deAl] Sn-USY (25) were chosen as reference materials to generate mesoporous catalysts. The nomenclature of catalysts used in this work lists the post-synthetic modifications in square brackets in the order in which they were applied, followed by the framework of the zeolites and the $\mathrm{Si} / \mathrm{Sn}$ content in round brackets. The $\mathrm{Sn}$ incorporation followed the dealumination step in all the procedures considered.

\subsection{Preparation of mesoporous Sn-Beta and Sn-USY catalysts}

The synthesis of mesoporous materials has a central role in heterogeneous catalysis [25]. Procedures can follow top-down or bottom-up approaches (Figure 2), both having their respective advantages and disadvantages $[16,26]$. Post-synthetic routes are usually preferred because of their ease and wide applicability. For this reason, emphasis in the current work was on the post-synthetic approach.

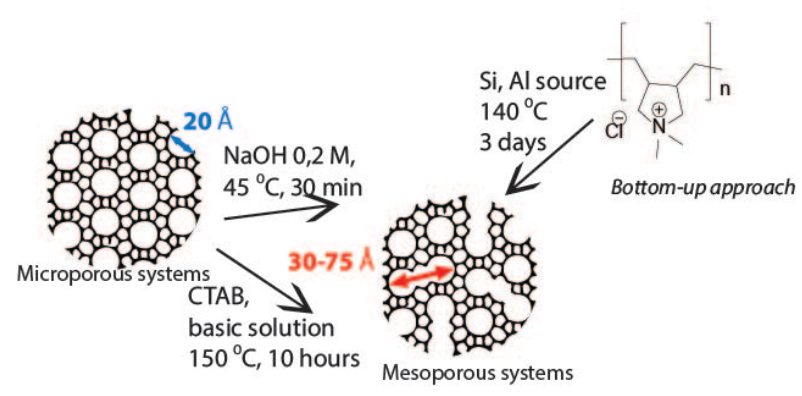

Figure 2. Schematic representation of bottom-up (right) and topdown (left) approaches for the preparation of mesoporous stannosilicates.

It is relatively easy to implement mesoporosity by desilication. The resulting mesoporous materials reduce diffusional limitations in zeolites, but this approach is difficult to control at the molecular level and leads to the formation of irregular porosity [27]. The desilication step was carried out on the commercial H-BEA zeolite followed by dealumination and $\mathrm{Sn}$ incorporation. As previously reported in the literature [19], the presence of aluminum was crucial for the formation of mesoporosity by alkaline treatment. The desilication of the [deAl] Sn-BEA caused a collapse of the crystalline structure as observed by XRD (Figure S2.2). H-USY zeolites showed some recalcitrance to the desilication process resulting in an inactive material. Recently, the use of surfactants in postsynthetic modification has been proposed [28], which involves dissolution of the zeolite in basic media and subsequent rearrangement or direct hydrothermal treatment. In both cases, reorganization of the structure and the formation of large and regular pores is facilitated by the presence of a surfactant. These procedures showed positive results starting from zeolites already containing Sn. Procedures involved the use of cetyltrimethylammonium bromide (CTAB), which was removed at the final stage by calcination. In this case, mesoporous surfactant-modified zeolites could be easily obtained for USY materials, while the preparation of BEA materials was not successful (Table 1).

Finally, a large-crystal mesoporous Sn-Beta was prepared using polydiallyl dimethylammonium chloride (PDADMA, $20 \mathrm{wt} \%$ ) together with tetraethyl ammonium hydroxide (TEAOH, $25 \mathrm{wt} \%$ ) as structure directing agents in a HF-free hydrothermal synthesis [29]. Table 1 lists the different synthetic procedures studied in this work.

Table 1. Catalyst synthesis methodologies

\begin{tabular}{|c|c|}
\hline Catalyst $^{\mathrm{a}}$ & Preparation ${ }^{b}$ \\
\hline $\begin{array}{l}\text { [deAl] Sn-BEA } \\
(100) /[\text { deAl] } \\
\text { Sn-USY }(25)\end{array}$ & $\begin{array}{l}\text { Acidic dealumination of } \\
\text { commercial H-Beta (19)/H- } \\
\text { USY (30) and Sn-impregnation } \\
{[23]}\end{array}$ \\
\hline $\begin{array}{l}\text { [deSi, deAl] Sn-BEA } \\
(100) /[\mathrm{deSi}, \mathrm{deAl}] \mathrm{Sn}- \\
\text { USY }(25)\end{array}$ & $\begin{array}{l}\text { Basic desilication, acidic } \\
\text { dealumination of commercial } \\
\text { H-Beta (19)/H-USY (30) and } \\
\text { Sn-impregnation [19] }\end{array}$ \\
\hline $\begin{array}{l}\text { [deAl, ST] Sn-BEA } \\
(100) / \\
{[\mathrm{deAl}, \mathrm{ST}] \mathrm{Sn}-\mathrm{USY}} \\
(25)\end{array}$ & $\begin{array}{l}\text { Surfactant templating of said } \\
\text { Sn containing zeolites [30] }\end{array}$ \\
\hline $\begin{array}{l}{[\mathrm{DR}, \text { deAl] Sn-USY }} \\
(25)\end{array}$ & $\begin{array}{l}\text { Dissolution and reassembly, } \\
\text { acidic dealumination of } \\
\text { commercial H-USY }(30) \text { and } \\
\text { Sn-impregnation }[31,32]\end{array}$ \\
\hline Sn-BEA HT (100) & $\begin{array}{l}\text { Hydrothermal synthesis using } \\
\text { TEAOH and PDADMA [29] }\end{array}$ \\
\hline
\end{tabular}

\subsection{Catalyst characterization}

Table 2 shows the characterization results of the synthesized crystalline materials. Procedures resulting in amorphous structures were omitted. The surfactant templating methodology required dissolution and 
reassembly in order to obtain mesoporous catalyst [DR, deAl] Sn-USY. As mentioned above, commercial USY zeolites are particularly resistant to post-synthetic modifications, as confirmed by the high aluminium content after acidic treatment and by the amorphous material obtained after basic desilication. The catalysts [deSi, deAl] Sn-USY (25) (basic desilication) and [deAl, ST] Sn-BEA (100) (surfactant templating) both lacked structural zeolite peaks in the XRD patterns, indicating amorphous materials. Therefore, these materials were not considered further (all XRD diffractograms are provided in Supporting Information S2). difficult and led to the formation of extra-framework metal oxides instead.

The absence of the characteristic $\mathrm{SnO}_{2}$ peaks in the XRD pattern of [deAl] Sn-USY (25) indicated amorphicity of these conglomerates or the presence of nanoparticle species too small to be visible by XRD. Notably, this catalyst presented a catalytic activity that was consistent with the other systems described herein (vide infra) without major drawbacks due to the presence of excessive extra-framework species.

Sn-Beta catalysts prepared by post-synthetic modifications present a different morphology than

\begin{tabular}{|c|c|c|}
\hline Catalyst & $\begin{array}{c}\text { Framework } \\
\text { structure }\end{array}$ & $\begin{array}{l}\text { BET surface } \\
\text { area }\left(\mathrm{m}^{2} / \mathrm{g}\right)^{\mathrm{a}}\end{array}$ \\
\hline $\begin{array}{l}{[\mathrm{deAl}] \mathrm{Sn}-\mathrm{BEA}} \\
(100)\end{array}$ & BEA & 592.4 \\
\hline $\begin{array}{l}\text { [deAl] Sn-USY } \\
(25)\end{array}$ & USY & 757.6 \\
\hline $\begin{array}{l}{[\mathrm{deSi}, \mathrm{deAl}]} \\
\text { Sn-BEA }(100)\end{array}$ & BEA & 637.7 \\
\hline $\begin{array}{l}{[\mathrm{deAl}, \mathrm{ST}] \mathrm{Sn}-} \\
\mathrm{USY}(25)\end{array}$ & USY & 539.7 \\
\hline $\begin{array}{l}{[\mathrm{DR}, \mathrm{deAl}] \mathrm{Sn}-} \\
\text { USY (25) }\end{array}$ & USY & 626.0 \\
\hline $\begin{array}{l}\text { Sn-BEA HT } \\
(100)\end{array}$ & BEA & 568.0 \\
\hline
\end{tabular}

The average pore sizes of the catalysts were measured by $\mathrm{N}_{2}$ adsorption and desorption analysis (Table 2), which confirmed a successful enlargement of the pores resulting in cavities with meso dimensions (30-75 $\AA)$. Using PDADMA as co-template in the hydrothermal synthesis, pore sizes with average dimensions of $70 \AA$ were obtained. The catalyst [deAl, ST] Sn-USY (25) did not show a significant enlargement of the porosity and thus was not investigated further.

SEM images of the synthesized catalysts in Figure 3 feature crystalline materials (additional SEM images are provided in Supporting Information Figure S3). Comparing the parental H-USY and the microporous [deAl] Sn-USY (25) (Figure 3d and e), it was evident that the dealumination procedure did not modify the morphology of the crystals (neither their shapes nor dimensions). However, small deposited clusters were visible on the surface of [deAl] Sn-USY (25) (Figure 4), suggesting that the stability of the commercial material made the incorporation of the $\mathrm{Sn}$

\begin{tabular}{cccc}
$\begin{array}{c}\text { Average pore } \\
\text { width }(\AA)^{\mathrm{a}}\end{array}$ & Si/Sn ratio & Si/Al ratio & $\begin{array}{c}\text { Acidity (mmol } \\
\left.\mathrm{NH}_{3} / \mathrm{g}\right)^{\mathrm{c}}\end{array}$ \\
\hline 21.8 & 102.5 & 134.8 & 0.071 \\
25.6 & 33.7 & 67.8 & 0.069 \\
48.0 & 129.0 & 137.0 & 0.095 \\
25.0 & 28.5 & 126.0 & - \\
34.0 & 52.0 & 70.0 & 0.058 \\
70.0 & 95.7 & 111.3 & 0.076
\end{tabular}

hydrothermally synthesized materials. Crystals of hydrothermal Sn-Beta zeolites prepared with the use of $\mathrm{HF}$ as mineralizing agent have a capped bipyramidal morphology [24]. In contrast, the textural properties strictly depend on the starting H-Beta zeolite in post-treated materials [11]. SEM pictures showed that during the $\mathrm{Sn}$ incorporation, the small crystals of the initial H-Beta zeolite reorganized into larger round-shape crystals (Supporting Information S3.1). The hydrothermally prepared material Sn-BEA HT (100) showed evident differences in appearance (Figure 3c). The crystals of the hydrothermal catalyst were the largest detected in the study, reaching dimensions of 2-2.5 $\mu \mathrm{m}$. SEM images also indicated the presence of amorphous material between the crystals, suggesting incomplete crystallization and a need for longer synthesis times in order to achieve complete crystal growth. Also here, absence of HF as a mineralizer led to the growth of spherical crystals.

The acidity of the synthesized materials was evaluated by $\mathrm{NH}_{3}-\mathrm{TPD}$, where comparable values of ammonia desorption $\left(0.08-0.13 \mathrm{mmol} \mathrm{NH}_{3} / \mathrm{g}\right)$ in all catalysts indicated similar acidity (Supporting Information S4) and the ability to compare activities 
based on varying porosities in the materials. The comparable acidity of the samples [deAl] Sn-USY and [DR, deAl] Sn-USY indicated catalytic inactivity of the extra-framework species in the [deAl] Sn-USY catalyst (Table 2).
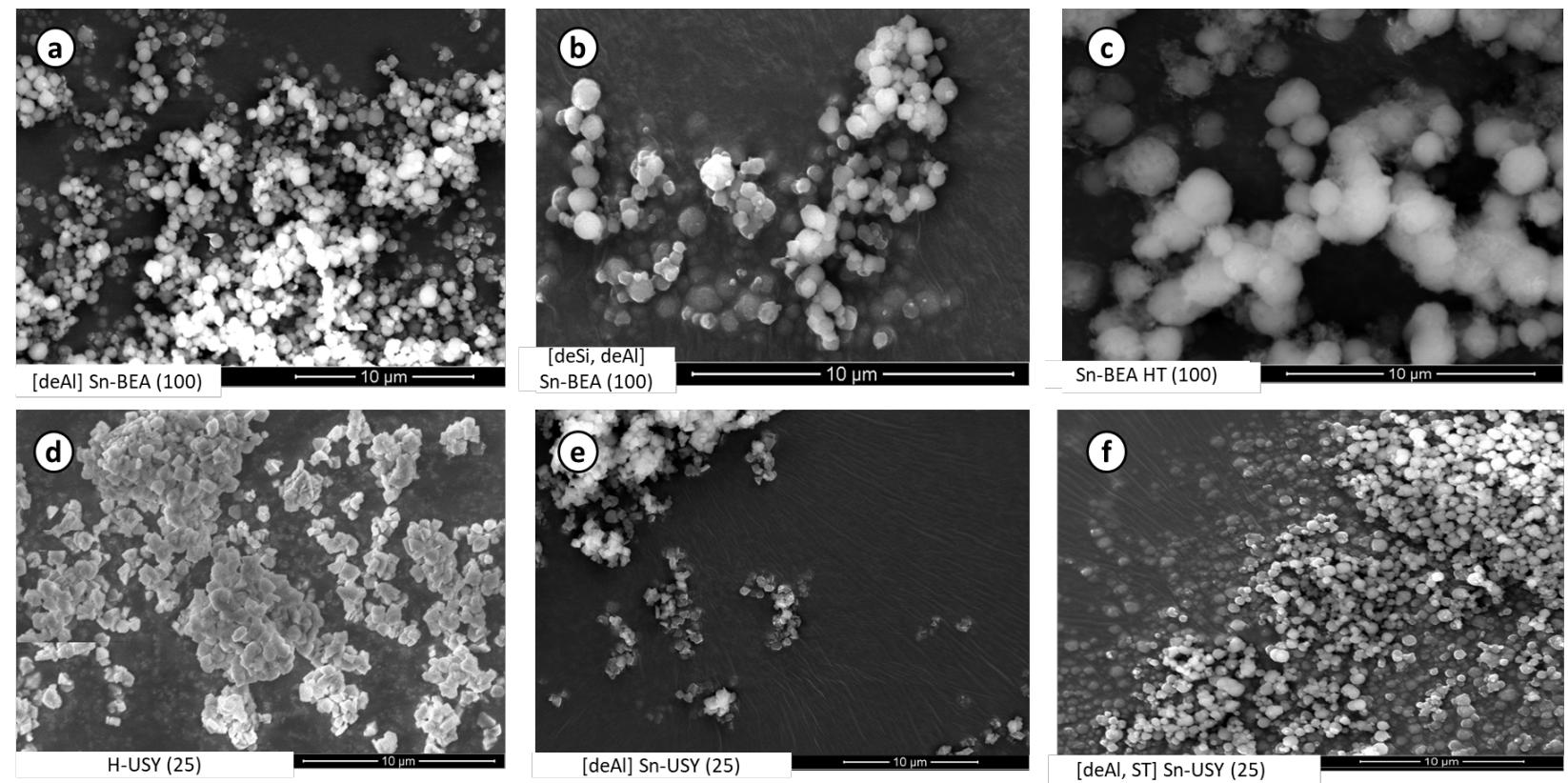

Figure 3. SEM images of microporous and mesoporous stannosilicates, (a) [deAl] Sn-BEA (100), (b) [deSi, deAl] Sn-BEA (100), (c) Sn-BEA HT (100), (d) [deAl] Sn-USY (25), (e) parental H-USY (6) and (f) [deAl, ST] Sn-USY (25).

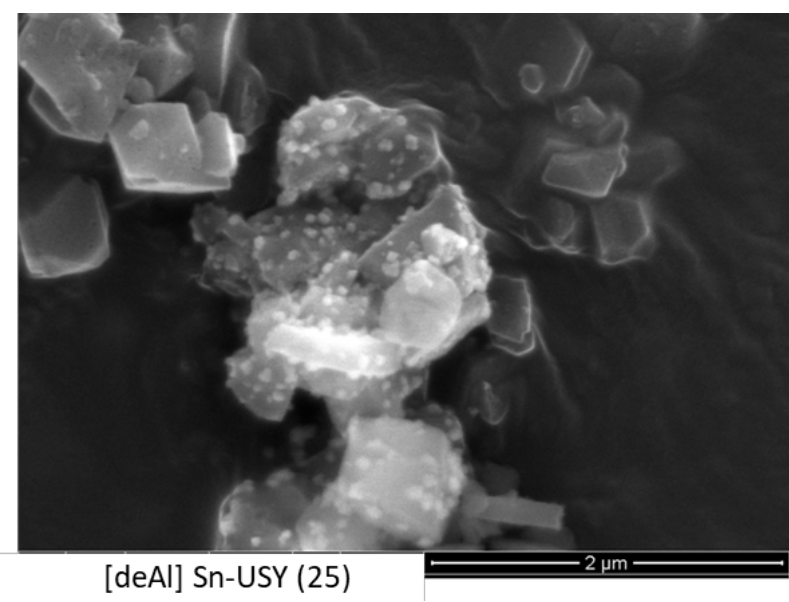

Figure 4. SEM image of microporous [deAl] Sn-USY, the picture shows the presence of extra-framework species

\subsection{Catalytic performance}

All prepared catalysts were tested for the conversion of carbohydrates with different sizes to methyl lactate in methanol at $160{ }^{\circ} \mathrm{C}$. Inulin, sucrose and glucose were used as substrates to evaluate the effect of mesoporosity and substrate size. The complete results of the catalytic tests are compiled in Supporting Information Figure S5. Inulins are storage carbohydrates in plants and contain $\beta(1-2)$ linked fructose monomers [33]. Due to its fairly easy degradation into monomeric fructose [34], inulin was chosen as substrate to investigate the benefits of mesoporous systems, without introducing major obstacles in the form of recalcitrance in an additional step of polymer solvolysis. 


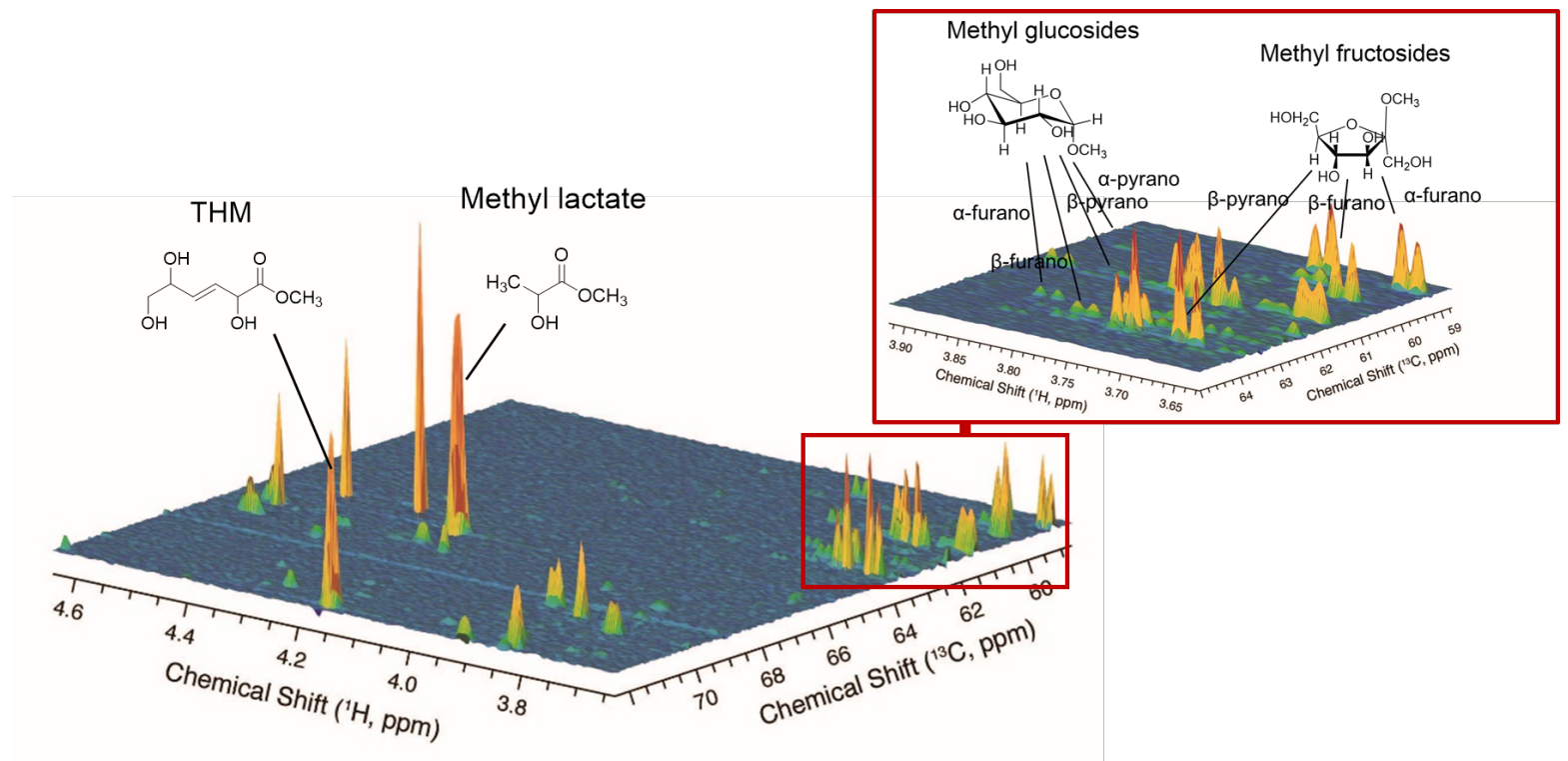

Figure 5. Identification of products in the reaction mixture using ${ }^{1} \mathrm{H}-{ }^{13} \mathrm{C}$ HSQC NMR spectra.

The crude reaction mixtures were analyzed by 2D NMR [35]. Particularly, ${ }^{1} \mathrm{H}-{ }^{13} \mathrm{C}$ HSQC allowed the distinction of the different sugars and methyl glycosides in their $\alpha / \beta$-anomeric and pyranoside/furanoside forms (Figure 5). Quantification of products was performed relative to internal quantification standards using ${ }^{13} \mathrm{C}$ NMR. Generally, the method allows accurate study of complex reaction mixtures without requiring purification, and in contrast to chromatographic techniques, calculation of response factors based on authentic standards is not required. Use of a quantitative ${ }^{13} \mathrm{C}$ NMR for the analysis of reaction mixtures obtained herein provides an accuracy in the determination of yields on the order of $0.5 \%[36,37]$.

In order to understand the catalytic properties of the different materials, the conversion of glucose, sucrose and inulin was studied by kinetic experiments conducted for up to $5 \mathrm{~min}$ for glucose and sucrose and for up to $15 \mathrm{~min}$ in the case of inulin (Figures 6, 7 and $8)$.
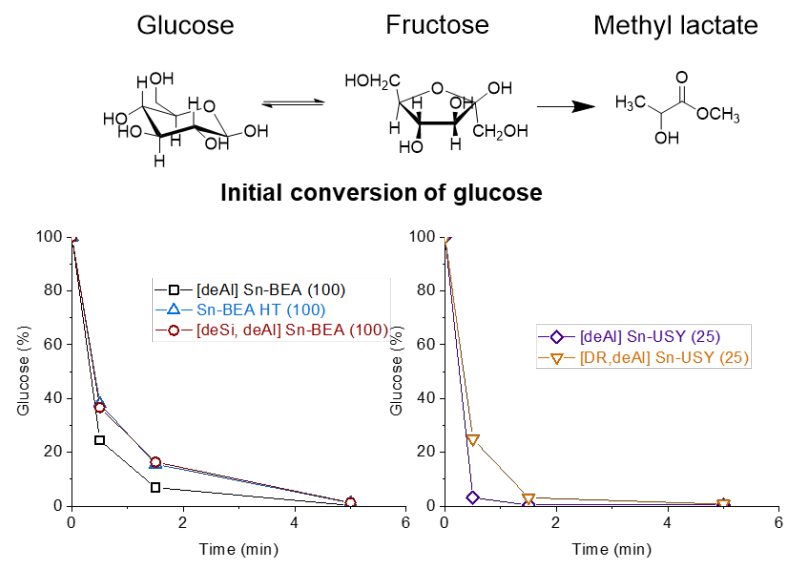

Initial formation of methyl lactate from glucose

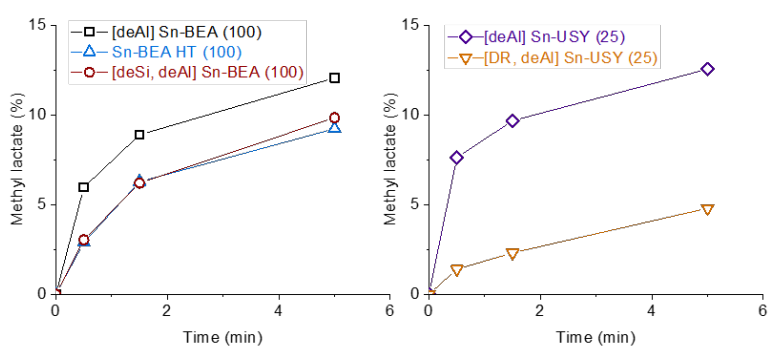

Figure 6. Short-time experiments on the conversion of glucose using different catalysts. Reaction conditions: glucose $3 \mathrm{wt} \%, 5 \mathrm{~mL}$ $\mathrm{MeOH}$, catalyst $1 \mathrm{wt} \%, 80 \mu \mathrm{L}$ DMSO as internal standard, $160{ }^{\circ} \mathrm{C}$, microwave reactor, yields based on qNMR analysis.

The mechanism for the conversion of glucose into methyl lactate involves the initial isomerization into fructose and subsequent retro-aldol cleavage and rearrangement into the final product [3]. In this case, the conventional microporous [deAl] Sn-BEA and 
[deAl] Sn-USY zeolites showed faster conversion of glucose and faster formation of methyl lactate than their mesoporous counterparts. These results suggested that glucose conversion to methyl lactate is not limited by diffusion in microporous catalysts and that the confinement effect or higher abundance of Sn sites in the micropores may positively influence the reaction (Figure 6).

When using sucrose as the substrate, the cleavage of the disaccharide by Brønsted acidity is required as a first step. This cleavage step complicates interpretation. However, the initial formation of methyl lactate was slightly faster also from sucrose substrate using the standard microporous catalysts as shown in Figure 7. The results also suggested that despite its size, sucrose conversion to methyl lactate is not diffusionally limited in strictly microporous materials.
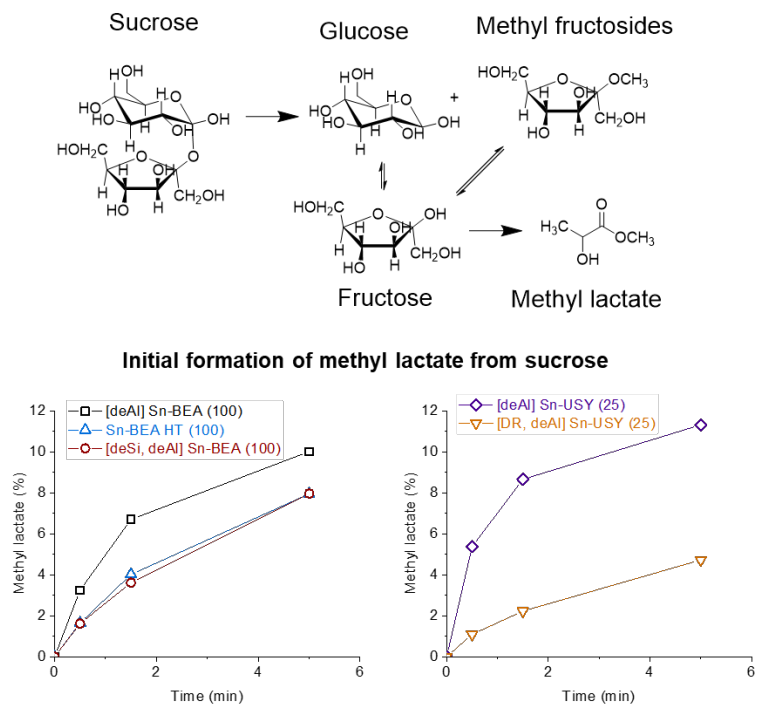

Figure 7. Short-time experiments on the conversion of sucrose using different catalysts. Reaction conditions: sucrose $3 \mathrm{wt} \%, 5 \mathrm{~mL}$ $\mathrm{MeOH}$, catalyst $1 \mathrm{wt} \%, 80 \mu \mathrm{L}$ DMSO as internal standard, $160{ }^{\circ} \mathrm{C}$, microwave reactor, yields based on qNMR analysis.

The time course for the conversion of inulin to methyl lactate is shown in Figure 8. The results exhibited some differences to the use of glucose and sucrose as substrates. In the conversion of inulin, mesoporous catalysts elicited faster formation both of methyl fructosides and of the final product methyl lactate (Figure 8) than microporous catalysts. The presence of larger pores thus appeared to facilitate the conversion of the largest sugar substrate employed in this work.

The initial rate of inulin conversion to methyl fructosides proceeded at different rates for the different catalytic systems. Both when using [deAl] Sn-BEA and [deAl] Sn-USY catalysts, the presence of mesopores led to faster initial solvolysis and formation of methyl fructosides, indicating less diffusional limitations for the bulky substrate to access the reaction pathway. In addition, the mesoporous BEA and USY catalysts also exhibited faster initial rates for the formation of methyl lactate.
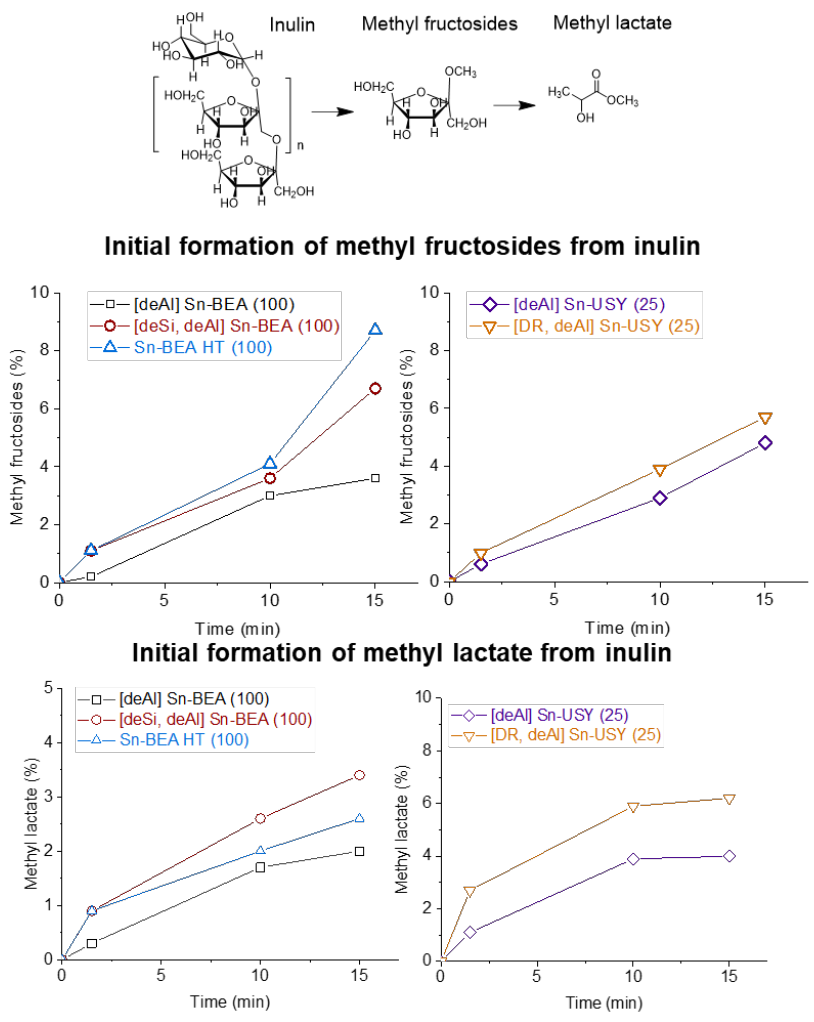

Figure 8. Short-time experiments on the conversion of inulin using different catalysts. Reaction conditions: inulin $3 \mathrm{wt} \%, 5 \mathrm{~mL} \mathrm{MeOH}$, catalyst $1 \mathrm{wt} \%, 80 \mu \mathrm{L}$ DMSO as internal standard, $160{ }^{\circ} \mathrm{C}$, microwave reactor, yields based on qNMR analysis.

It is noticeable that formation of methyl fructosides and of methyl lactate was slower when increasing the size of the substrate regardless of the catalyst used. These results are consistent with the increasing numbers of reaction steps needed to generate monosaccharides and methyl lactate from the different sugars. All catalysts tested herein formed negligible amount of methyl glucosides and of furanic byproducts, indicating only minor Brønsted acidity in the synthesized stannosilicate catalysts.

For inulin conversion, Figure 9 shows that the mesoporous catalysts did not achieve improved yields of methyl lactate compared to the parent microporous systems for USY zeolites and only a slight improvement in the case of the BEA framework. However, the yields at full conversion are not 
appropriate indicators of changes in catalytic activity. In fact, methyl fructosides are considered intermediate products in the process [35], which easily hydrolyze in the presence of water to yield fructose as a substrate for the formation of methyl lactate (or other bio-based chemicals) on a longer time scale [38]. All the modified systems showed increased production of these intermediates and a little change to the formation of the desired product. Therefore, longer raction times are necessary to convert all the intermediates formed from inulin and mesoporosity does not drastically affect the overall yields in methyl lactate at full conversion. Hence, the average pore size was not a limiting factor for the methyl lactate yields starting from inulin in batch reactions. The reactivity of the catalytic systems was strictly correlated to the Lewis acidity of the incorporated $\mathrm{Sn}$ and diffusional limitations can be overcome by changing the reaction parameters, especially by using prolonged reaction times.

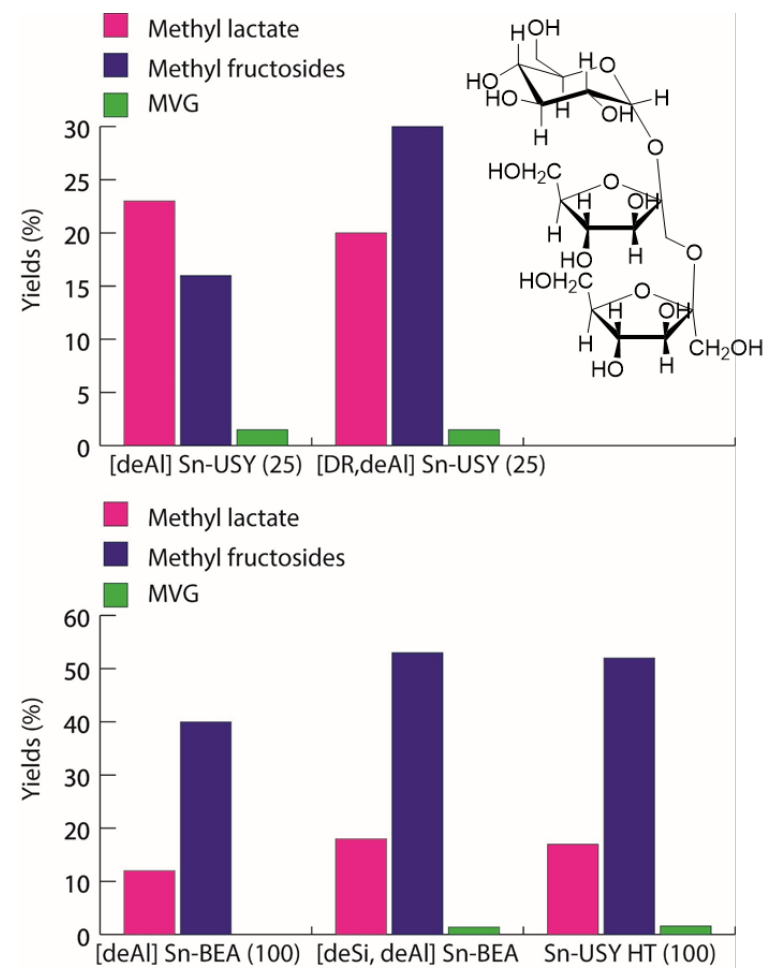

Figure 9. Conversion of inulin using different catalytic systems (methyl fructosides can be considered intermediates that yield methyl lactate at long reaction times). Reaction conditions: inulin 3 $\mathrm{wt} \%, 5 \mathrm{~mL} \mathrm{MeOH}$, catalyst $1 \mathrm{wt} \%, 80 \mu \mathrm{L}$ DMSO as internal standard, $160{ }^{\circ} \mathrm{C}, 4 \mathrm{~h}$, microwave reactor, yields based on qNMR analysis.

The yields of methyl lactate normalized by the Sn-content are listed in the Supporting Information S7. The values highlighted the results described previously; large pore materials favoured the formation of methyl lactate in the case of inulin as substrate, whereas the mesoporosity did not affect greatly the yield when using glucose or sucrose as starting reagents. Moreover, the normalized yields allowed to compare the performance of the USY (25) and BEA (100) frameworks, which was slightly better for the Sn BEA zeolites. This result correlated well with the formation of inactive Sn oxide species on the surface of Sn USY zeolites, as observed by SEM.

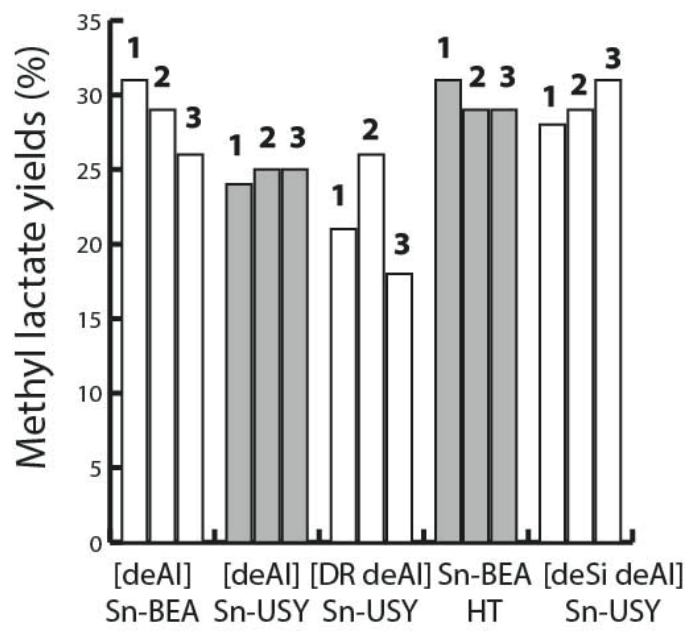

Figure 10. Methyl lactate yields during three consecutive catalysts runs. Reaction conditions: glucose $3 \mathrm{wt} \%, 5 \mathrm{~mL} \mathrm{MeOH}$, catalyst 1 wt $\%, 80 \mu \mathrm{L}$ DMSO as internal standard, $160{ }^{\circ} \mathrm{C}, 2 \mathrm{~h}$, microwave reactor, yields based on $\mathrm{qNMR}$ analysis.

The possibility of recycling and reusing the catalysts was studied by carrying out three consecutive catalytic runs without intermediate regeneration and using glucose as starting substrate. Overall, the studied zeolites exhibited high stability (Figure 10), where only [deAl] Sn-BEA (100) showed a slight decrease in the catalytic activity in consecutive runs. The increase in methyl lactate yields after recycling the Sn-USY catalysts could be due to the removal of inactive extraframework $\mathrm{SnO}_{2}$ during the first experiment.

\section{Conclusions}

Different zeolite structures were tested in the preparation of mesoporous stannosilicate catalysts for the conversion of different sized sugars into methyl lactate. After a preliminary screening, the frameworks USY and BEA were selected for post-synthetic modifications involving removal of $\mathrm{Al}$ and incorporation of Sn. Different procedures to introduce mesoporosity within the zeolite structures were evaluated with an emphasis on post-synthetic modifications that provide low degree of control, but easy execution and possibility for scaling-up. Basic desilication was only successful with the BEA 
framework, leading to a complete structural collapse for USY zeolites. Methodologies using surfactant templating resulted in active mesoporous material for the zeolite USY in the case of the dissolutionreassembly procedure. Finally, a hierarchical Sn-Beta zeolite was prepared by hydrothermal synthesis of the mesoporous Beta zeolite, dealumination and $\mathrm{Sn}$ incorporation.

The most promising mesoporous Sncontaining zeolites were obtained by basic desilication and acidic dealumination of commercial H-BEA as well as dissolution, reassembly and acidic dealumination of commercial H-USY. Different prepared stannosilicates were tested as catalysts for the formation of methyl lactate from simple sugars and from the oligomer inulin. The experiments were carried out at full substrate conversion and indicated that all materials retained similar selectivity in the formation of methyl lactate. Lower diffusional limitations in mesoporous catalysts resulted in a higher initial rate of methyl lactate formation from the largest substrate inulin. In contrast, conversion of glucose and sucrose to methyl lactate was slower over the mesoporous catalysts, suggesting an impact of confinement in the conversion of these smaller sugars.

\section{Experimental}

\subsection{Materials}

Glucose (99.5\%), sucrose (99.5\%), inulin, cellobiose (98\%), maltose, nitric acid (>65\%), $\mathrm{SnCl}_{4}$ pentahydrate $(98 \%)$, tetraethylammoium hydroxide (TEAOH, 35\% water), cetyltrimethyl ammonium bromide (CTAB, 98\%), polydiallyl dimethylammonium chloride (PDADMA, $20 \mathrm{wt} \%$ ), $\mathrm{NH}_{4} \mathrm{OH}(50 \% \mathrm{w} / \mathrm{w})$, methanol (99.8\%, anhydrous), methanol-d4 (99.8\%), NaOH and dimethyl sulfoxide (DMSO, >99.5\%) were purchased from SigmaAldrich and used as recieved. $\mathrm{NH}_{4}{ }^{+}$-zeolites were obtained from Zeolyst International and calcined at $550{ }^{\circ} \mathrm{C}$ for $6 \mathrm{~h}$ in order to obtain the $\mathrm{H}$-forms of the zeolites. $\mathrm{NaAlO}_{2}$ and fumed silica were acquired from Redel-de Haën and used without any further treatment.

\subsection{General procedure for the preparation of post-synthetic stannosilicates}

Stannosilicates were prepared by post-synthesis following a two-step procedure [23]. First, acidic dealumination was performed in nitric acid (13 M, 20 $\left.\mathrm{mL} \mathrm{g} \mathrm{g}^{-1}, 100{ }^{\circ} \mathrm{C}, 20 \mathrm{~h}\right)$. The resulting zeolite was filtered, washed with deionized water until the washing water had a neutral $\mathrm{pH}$ and dried at $120{ }^{\circ} \mathrm{C}$ overnight. The Sn was then incorporated by incipient wetness impregnation of aqueous solutions of $\mathrm{SnCl}_{4} \cdot 5 \mathrm{H}_{2} \mathrm{O}$, and the final material calcined at $550{ }^{\circ} \mathrm{C}$ for $6 \mathrm{~h}$.

\subsection{Procedure of desilication}

The basic desilication was carried out in $0.2 \mathrm{M} \mathrm{NaOH}$, $30 \mathrm{~mL} \mathrm{~g}{ }^{-1}, 45{ }^{\circ} \mathrm{C}$ for $30 \mathrm{~min}$. The desilicated zeolite was then washed until neutral $\mathrm{pH}$ of the washing water, dried overnight at $120^{\circ} \mathrm{C}$ and used as starting material for the preparation of stannosilicates [19].

\subsection{Surfactant templating top-down synthesis}

$2.5 \mathrm{~g}$ of Sn-zeolite were added to a solution of $1.75 \mathrm{~g}$ of cetyltrimethylammonium bromide (CTAB) in 160 $\mathrm{mL}$ of $0.37 \mathrm{M} \mathrm{NH}_{4} \mathrm{OH}$, and the mixture stirred for 20 min and transferred to a Teflon-lined stainless steel autoclave. After hydrothermal treatment at $150{ }^{\circ} \mathrm{C}$ for $10 \mathrm{~h}$ the solid was filtered, washed with water, dried overnight at $70{ }^{\circ} \mathrm{C}$ and calcined at $550{ }^{\circ} \mathrm{C}$ for $6 \mathrm{~h}$. The procedure followed the method described in [30].

\subsection{Dissolution-reassembly top-down synthesis}

$1 \mathrm{~g}$ of commercial H-zeolite was suspended in $64 \mathrm{~mL}$ of $0.36 \mathrm{M} \mathrm{NH}_{4} \mathrm{OH}$. After $2 \mathrm{~h}$ of stirring, $0.7 \mathrm{~g}$ of CTAB was added and the final solution transferred to a Teflon-lined stainless steel autoclave for the hydrothermal treatment at $150{ }^{\circ} \mathrm{C}$ for $48 \mathrm{~h}$. The solid was then filtered, washed, dried and calcined [31].

\subsection{Hydrothermal synthesis of mesoporous Beta zeolite}

A solution of $0.16 \mathrm{~g}$ of $\mathrm{NaOH}$ and $0.3 \mathrm{~g}$ of $\mathrm{NaAlO}_{2}$ in $32 \mathrm{~mL}$ of TEAOH was prepared, then $4.8 \mathrm{~g}$ of fumed silica were added, and the mixture gently stirred until a homogeneous gel was obtained. Subsequently, $3.0 \mathrm{~g}$ of PDADMA were added and the resulting mixture stirred for $24 \mathrm{~h}$ and transferred to a Teflon-lined stainless steel autoclave for hydrothermal crystallization during one week at $140{ }^{\circ} \mathrm{C}$ [29]. The final material was used as starting reagent for the preparation of stannosilicates, as specified above.

\subsection{Catalyst characterization}

The amounts of silicon, tin and aluminium in samples were determined by X-ray fluorescence (XRF) using a PANalytical epsilon3-XL. The Si/Al and $\mathrm{Si} / \mathrm{Sn}$ ratios were obtained from calibration curves.

BET surface areas and pore dimensions were obtained from $\mathrm{N}_{2}$ physisorption measurements using a 
Micrometrics ASAP 2020 surface area and porosity analyzer. Samples were initially degassed at $200{ }^{\circ} \mathrm{C}$ in vacuum for $4 \mathrm{~h}$ and adsorption-desorption isotherms subsequently acquired at $-196^{\circ} \mathrm{C}$.

Power X-ray diffraction (XRD) patterns of samples were acquired on a Huber G670 imagingplate Guinier powder-diffraction camera with $\mathrm{CuK} \alpha$ radiation $(\lambda=0.154184 \mathrm{~nm})$ and recorded in the $2 \theta$ range from 3 to $50^{\circ}$.

Scanning electron microscopy (SEM) images were recorded on a FEI Quanta 200 ESEM FEG microscope with using an accelerating voltage of $5 \mathrm{kV}$.

The acidity of the zeolites was characterized by ammonia temperature programmed desorption ( $\left.\mathrm{NH}_{3}-\mathrm{TPD}\right)$ using an Autochem II Chemisorption analyzer. The samples were pretreated at $550{ }^{\circ} \mathrm{C}$, and the adsorption carried out in $10 \% \mathrm{NH}_{3}$ in $\mathrm{He}$ flow for 15 min at $150{ }^{\circ} \mathrm{C}$. The desorption was done using a temperature ramp of $10^{\circ} \mathrm{C} / \mathrm{min}$ from 150 to $550^{\circ} \mathrm{C}$.

\subsection{General procedure for the catalytic experiments}

In a typical reaction, $120 \mathrm{mg}$ of substrate were reacted in $5 \mathrm{~mL}$ of methanol with $50 \mathrm{mg}$ of catalyst and $80 \mu \mathrm{L}$ of dimethyl sulfoxide (DMSO) as internal standard in a microwave reactor at $160{ }^{\circ} \mathrm{C}$. Experiments at full conversion were carried out with reaction times of $2 \mathrm{~h}$, while variable times were used for the initial-rate experiments. After the reaction, the solution was filtered using a $0.22 \mu \mathrm{m}$ Nylon Frisenette syringe filter and the crude mixture analyzed by NMR.

For a catalyst recycle study, the reaction mixture was filtered under vacuum and the recovered catalyst washed extensively with methanol and dried at $120^{\circ} \mathrm{C}$ for 30 minutes. Subsequently, the recovered zeolite was directly used for the next catalytic run.

\subsection{Analysis of the product mixtures}

The crude reaction mixtures were analysed by NMR using a Bruker AVANCE III $400 \mathrm{MHz}$ equipped with a CryoProbe Prodigy and a Bruker AVANCE III 800 $\mathrm{MHz}$ with a TCI CryoProbe, both instruments equipped with automatic samples changers. NMR samples were prepared by addition of $100 \mu \mathrm{L}$ of d4methanol as lock substance to $500 \mu \mathrm{L}$ of crude reaction, carried out in protonated methanol as solvent. Yields of products were based on quantitative ${ }^{13} \mathrm{C}$ NMR analysis. Calculations were performed by comparison with the intensity of the peak of the
DMSO present as internal standard, following the formula:

$[\mathrm{X}]=[\mathrm{DMSO}]\left(\mathrm{nC}_{(\mathrm{DMSO})} / \mathrm{nC}_{(\mathrm{x})}\right)\left(\mathrm{A}_{(\mathrm{x})} / \mathrm{A}_{(\mathrm{DMSO})}\right)$

where $[\mathrm{X}]$ and $[\mathrm{DMSO}]$ are the concentrations of the product $\mathrm{X}$ and $\mathrm{DMSO}$, respectively, $\mathrm{nC}$ are the number of carbons generating the signal (2 for DMSO), and $\mathrm{A}_{(\mathrm{x})}$ and $\mathrm{A}_{(\mathrm{DMSO})}$ the integrated areas of the selected signals.

Sugars in the reaction were studied by ${ }^{1} \mathrm{H}-{ }^{13} \mathrm{C}$ HSQC in the spectral region of the primary alcohols, acquiring a spectral width of $30 \mathrm{ppm}$, with a frequency offset of $62 \mathrm{ppm}$ in the carbon dimension. Carbohydrates were analyzed quantitatively by ${ }^{1} \mathrm{H}-{ }^{13} \mathrm{C}$ HSQC after the construction of calibration curves for both the 400 and $800 \mathrm{MHz}$ instruments.

\section{Acknowledgements}

This work was funded by the Innovation Fund Denmark (case number 5150-00023B). $800 \mathrm{MHz}$ NMR spectra were recorded by using the spectrometer of the NMR center DTU supported by the Villum foundation. AS acknowledges the Erasmus + Programme for the traineeship grant.

\section{References}

1. Sudarsanam, P., et al., Functionalised heterogeneous catalysts for sustainable biomass valorisation. Chemical Society Reviews, 2018.

2. Nemeth, L. and S.R. Bare, Chapter One Science and Technology of Framework Metal-Containing Zeotype Catalysts, in Advances in Catalysis, F.C. Jentoft, Editor. 2014, Academic Press. p. 1-97.

3. Holm, M.S., S. Saravanamurugan, and E. Taarning, Conversion of Sugars to Lactic Acid Derivatives Using Heterogeneous Zeotype Catalysts. Science, 2010. 328(5978): p. 602-605.

4. Moliner, M., Y. Román-Leshkov, and M.E. Davis, Tin-containing zeolites are highly active catalysts for the isomerization of glucose in water. Proceedings of the National Academy of Sciences, 2010. 107(14): p. 6164-6168.

5. Román-Leshkov, Y., et al., Mechanism of Glucose Isomerization Using a Solid Lewis Acid Catalyst in Water. Angewandte Chemie International Edition, 2010. 49(47): p. 8954- 
8957.

6. Bermejo-Deval, R., et al., Active Sites in SnBeta for Glucose Isomerization to Fructose and Epimerization to Mannose. ACS Catalysis, 2014. 4(7): p. 2288-2297.

7. Tolborg, S., et al., Tin-containing silicates: identification of a glycolytic pathway via 3deoxyglucosone. Green Chemistry, 2016. 18(11): p. 3360-3369.

8. Mäki-Arvela, P., et al., Production of Lactic Acid/Lactates from Biomass and Their Catalytic Transformations to Commodities. Chemical Reviews, 2014. 114(3): p. 19091971.

9. European Bioplastics e.V. Bioplastics market data. 2018; Available from: https://www.european-

bioplastics.org/market/.

10. Elliot, S.G., et al., Synthesis of a novel polyester building block from pentoses by tincontaining silicates. RSC Advances, 2017. 7(2): p. 985-996.

11. van der Graaff, W.N.P., et al., Influence of pore topology on synthesis and reactivity of Sn-modified zeolite catalysts for carbohydrate conversions. Catalysis Science \& Technology, 2017. 7(14): p. 3151-3162.

12. Elliot, S.G., et al., NMR Spectroscopic Isotope Tracking Reveals Cascade Steps in Carbohydrate Conversion by Tin-Beta. ChemCatChem, 2018. 10(6): p. 1414-1419.

13. De Clercq, R., M. Dusselier, and B.F. Sels, Heterogeneous catalysis for bio-based polyester monomers from cellulosic biomass: advances, challenges and prospects. Green Chemistry, 2017. 19(21): p. 5012-5040.

14. De Clercq, R., et al., Confinement Effects in Lewis Acid-Catalyzed Sugar Conversion: Steering Toward Functional Polyester Building Blocks. ACS Catalysis, 2015. 5(10): p. 5803-5811.

15. Schwieger, W., et al., Hierarchy concepts: classification and preparation strategies for zeolite containing materials with hierarchical porosity. Chemical Society Reviews, 2016. 45(12): p. 3353-3376.

16. Serrano, D.P., J.M. Escola, and P. Pizarro, Synthesis strategies in the search for hierarchical zeolites. Chemical Society Reviews, 2013. 42(9): p. 4004-4035.

17. Silaghi, M.-C., C. Chizallet, and P. Raybaud, Challenges on molecular aspects of dealumination and desilication of zeolites. Microporous and Mesoporous Materials, 2014. 191: p. 82-96.

18. Holm, M.S., et al., Catalysis with hierarchical zeolites. Catalysis Today, 2011. 168(1): p. 3-16.

19. Al-Nayili, A., K. Yakabi, and C. Hammond, Hierarchically porous BEA stannosilicates as unique catalysts for bulky ketone conversion and continuous operation. Journal of Materials Chemistry A, 2016. 4(4): p. 1373-1382.

20. Jin, J., et al., Synthesis of mesoporous Beta and Sn-Beta zeolites and their catalytic performances. Dalton Transactions, 2014. 43(22): p. 8196-8204.

21. Yao, J.-K., et al., Hierarchically porous $S n-\beta$ zeolites via an OSDA-free synthesis. Green Chemistry, 2017. 19(14): p. 3214-3218.

22. Zhang, J., et al., Hierarchical Sn-Beta Zeolite Catalyst for the Conversion of Sugars to Alkyl Lactates. ACS Sustainable Chemistry \& Engineering, 2017. 5(4): p. 3123-3131.

23. Hammond, C., S. Conrad, and I. Hermans, Simple and scalable preparation of highly active Lewis acidic Sn-beta. Angew Chem Int Ed Engl, 2012. 51(47): p. 11736-9.

24. Tolborg, S., et al., Incorporation of tin affects crystallization, morphology, and crystal composition of Sn-Beta. Journal of Materials Chemistry A, 2014. 2(47): p. 20252-20262.

25. Roth, W.J. and J.C. Vartuli, Synthesis of mesoporous molecular sieves, in Studies in Surface Science and Catalysis, J. Cejka and H. van Bekkum, Editors. 2005, Elsevier. p. 91-110.

26. Surfactant-Templated Mesostructuring of Zeolites: From Discovery to Commercialization, in Mesoporous Zeolites.

27. Verboekend, D. and J. Pérez-Ramírez, Design of hierarchical zeolite catalysts by desilication. Catalysis Science \& Technology, 2011. 1(6): p. 879-890.

28. Sachse, A. and J. García-Martínez, Surfactant-Templating of Zeolites: From Design to Application. Chemistry of Materials, 2017. 29(9): p. 3827-3853.

29. Wang, L., et al., Hierarchical mesoporous zeolites with controllable mesoporosity templated from cationic polymers. Microporous and Mesoporous Materials, 2010. 131(1): p. 58-67. 
30. García-Martínez, J., et al., Mesostructured zeolite $Y$-high hydrothermal stability and superior FCC catalytic performance. Catalysis Science \& Technology, 2012. 2(5): p. 987-994.

31. Goto, Y., et al., Mesoporous Material from Zeolite. Journal of Porous Materials, 2002. 9(1): p. 43-48.

32. Ivanova, I.I. and E.E. Knyazeva, Micromesoporous materials obtained by zeolite recrystallization: synthesis, characterization and catalytic applications. Chemical Society Reviews, 2013. 42(9): p. 3671-3688.

33. Barclay, T., et al., Analysis of the hydrolysis of inulin using real time $1 H$ NMR spectroscopy. Carbohydrate Research, 2012. 352: p. 117-125.

34. Abasaeed, A.E. and Y.Y. Lee, Inulin hydrolysis to fructose by a novel catalyst. Chemical Engineering \& Technology, 1995. 18(6): p. 440-444.

35. Tosi, I., et al., Kinetic analysis of hexose conversion to methyl lactate by Sn-Beta: effects of substrate masking and of water. Catalysis Science \& Technology, 2018. 8(8): p. 2137-2145.

36. Elliot, S.G., et al., Quantitative NMR Approach to Optimize the Formation of Chemical Building Blocks from Abundant Carbohydrates. ChemSusChem, 2017. 10(14): p. 2990-2996.

37. Rundlof, T., et al., Survey and qualification of internal standards for quantification by $1 \mathrm{H}$ NMR spectroscopy. J Pharm Biomed Anal, 2010. 52(5): p. 645-51.

38. Saravanamurugan, S., et al., Facile and benign conversion of sucrose to fructose using zeolites with balanced Brønsted and

Lewis acidity. Catalysis Science \& Technology, 2017. 7(13): p. 2782-2788. 